\title{
THE INFLUENCE OF NONLINEAR AND LINEAR DEFECTS ON THE LIGHT PROPAGATION THROUGH LINEAR ONE-DIMENSIONAL PHOTONIC LATTICE
}

\section{Slavica Kuzmanovićc ${ }^{*}$, Marija Stojanović Krasić ${ }^{2}$, Ana Mančić ${ }^{3}$, Branko Drljača ${ }^{1}$ and Milutin Stepić ${ }^{4}$}

${ }^{1}$ Faculty of Natural Sciences and Mathematics, University of Priština, Kosovska Mitrovica, Serbia.

${ }^{2}$ Faculty of Technology, University of Niš, Leskovac, Serbia.

${ }^{3}$ Faculty of Natural Sciences and Mathematics, University of Niš, Niš, Serbia.

${ }^{4}$ Vinča Institute of Nuclear Sciences, University of Belgrade, Belgrade, Serbia.

\begin{abstract}
In this paper, the light beam propagation through one-dimensional photonic lattice, possessing one nonlinear defect and one linear defect, has been investigated numerically. Different dynamical regimes have been identified in terms of the distance between the two defects, position of the incident light beam, the width of linear defect, the values of nonlinearity and presence of the transverse kick. Strong localized modes on the defects, breathing and zig-zag modes in the area between defects have been observed. It has been concluded that the width of the linear defect placed

next to the nonlinear one influences localization of the beam at the nonlinear waveguide. On the other hand, the nonlinear defect, regardless of the values of nonlinearity, have a small influence on the beam propagation in photonic lattice. It has been observed that the transverse kick of the initial beam leads to the distortion of localized structures. By launching the light beam towards defects, the reflection of light has been noticed. Presented results can be useful for different applications, such as blocking, filtering and routing of light beam through optical media.
\end{abstract}

Key words: waveguide array, nonlinear defect, linear defect, light localization, control of light propagation.

\section{INTRODUCTION}

Photonic lattices (PL) represent special kind of photonic crystals which consist of periodic arrays of waveguides (WGs) closely spaced to provide weak coupling between neighboring WGs through their evanescent fields. PLs offer the possibility to control the light beam by changing the system parameters, such as refractive index and period of the lattice. Due to their properties and structure, they represent suitable systems for investigation of the wave propagation in periodic systems (Denz et all., 2010) and visualization of different effects such as discrete diffraction (Christodoulides et al., 1988), Fano resonance ((Miroshnichenko et all., 2010), (Naether et al., 2009)), Anderson localization ((Lahini et al., 2008), (Schwartz et al., 2007)), etc. In PLs, different defects can appear in the process of their fabrication, but nowadays, they can be made intentionally and used in attempts to control the light beam propagation ((Noda et al., 2007), (Tran, 1997)). Defects in PL can be formed by changing the value of refractive index in certain WG or by changing the width of the WG or the distance between WGs ((Meier et al., 2005), (Morandotti et al., 2003)). These defects destroy the translation symmetry of the system but, at the same time, they enable the existence of different stable, localized modes ((Beličev et al., 2010), (Fedele et al., 2005), (Molina et al., 2008)). Recently, the influence of nonlinear defect (ND) on the light beam propagation in one-dimensional (1D) linear uniformed lattice has been investigated (Kuzmanović et al., 2015a) as well as the influence of linear defect (LD) and interface defect on the beam dynamics (Stojanović Krasić et al., 2016).

In this work, we investigate numerically light beam propagation through uniform, 1D PL possessing one linear and one nonlinear defect. The paper is organized in the following way. Mathematical model of wave propagation through the system is formulated in Section 2. By changing the initial position of the beam with respect to the position of the ND and LD, 
the width of LD and incident angle of the light beam, different regimes have been obtained and these numerical results are presented and discussed in Section 3. In Section 4, the conclusions have been briefly summarized.

\section{MODEL EQATIONS}

We consider the PL which consists of the linear WG arrays with embedded one nonlinear WG (i. e. nonlinear defect) and one linear defect (Figure 1).

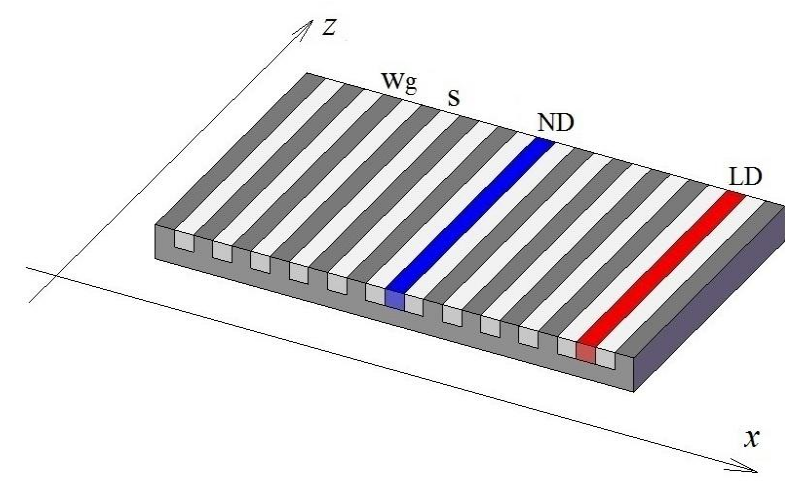

Figure 1. Schematic representation of the system. Blue line shows the position of the nonlinear defect, while the red line denotes the position of the linear defect.

Light propagation in the lattice has been modelled by the paraxial time-independent Helmholtz equation (Kuzmanović et al., 2015a):

$$
i \frac{\partial E}{\partial z}+\frac{1}{2 k_{0} n_{0}} \frac{\partial^{2} E}{\partial x^{2}}+k_{0} n_{0} n(x) E=0,
$$

where $z$ is the propagation amplitude, $E(x, z)$ represents the component of the light electric field in the $z$-direction, $k_{0}=2 \pi / \lambda$ is the wave number, $n_{0}$ is the refractive index of the substrate, whereas $\lambda$ marks the wavelength of the incident beam. The lattice contains 49 WGs on the left and 49 WGs on the right of ND in the $x$ (transverse) direction. The stationary profile of refractive index of the lattice is given by the following equation:

$$
n(x)=n_{l}(x)+n_{n l}(x),
$$

where $n_{l}(x)$ represents the linear part of the refractive index and $n_{n l}(x)$ is local nonlinear term. Linear part of the refractive index is defined in a form:

$$
n_{l}(x)=\Delta n\left(\begin{array}{l}
\sum_{j=1}^{k-1} G_{j}\left(w_{g}, s, x\right)+G_{k}\left(w_{g k}, s_{k}, x\right) \\
+\sum_{j=k+1}^{n} G_{j}\left(w_{g}, s, x\right)
\end{array}\right),
$$

where $\Delta n$ is the value of lattice potential, $k$ is the position of the LD which is arbitrary placed in the lattice, $N$ is the total number of WGs in the lattice. Parameter $w_{g}$ marks the width of the WGs, whereas $w_{g k}$ represents the width of the LD. Functions $G_{j}\left(w_{g}, s, x\right)$ represent Gaussians corresponding to the lattice WGs, whereas function $G_{k}\left(w_{g k}, s_{k}, x\right)$ corresponds to the LD.

Nonlinear part of the refractive index locally induced at one WG is defined in a form:

$$
n_{n l}(x)=-\frac{1}{2} n_{0}^{2} r E_{p v} \frac{|E|^{2}}{I_{d}+|E|^{2}} \delta,
$$

where $E_{p v}$ is component of the light electric field, $r$ is electro-optic coefficient, $\delta$ is the Kronecker delta symbol and $I_{d}$ is dark irradiance ((Chen et al., 2005), (Smirnov et al., 2006)). Dark irradiance is the parameter of the material proportional to the number of thermally generated photons in non-lighted material.

Introducing dimensionless variables $\zeta=k_{0} x$ and $\eta=k_{0} z$, the equation (1) can be written in the following dimensionless form:

$$
i \frac{\partial E}{\partial \eta}+\frac{1}{2 n_{0}} \frac{\partial^{2} E}{\partial \zeta^{2}}+n_{0} n(\zeta) E=0 .
$$

The light propagation across the lattice is initiated by the Gaussian shaped incident beam with the FWHM of the order of the width of the lattice WGs, and simulated numerically by the split - step Fourier method (Radosavljević et all., 2014). The intensity of the incident light beam, with a wavelength $\lambda=514.5 \mathrm{~nm}$ is kept at fixed value, whereas the position of the injection with respect to the defect position, its transverse tilt $\alpha$ and the nonlinearity and the width of LD are changeable parameters. 


\section{RESULTS AND DISCUSSION}

In the PL with ND and LD, qualitatively different dynamical behaviour has been observed in comparison to the previously investigated cases with one ND located in the uniform lattice (Kuzmanović et al., 2015a) or with one geometrical defect and LD in the composite system (Kuzmanović et al., 2015b). The width of the LD is either 2 or $6 \mu \mathrm{m}$ and further in the paper they will be marked as narrow and wide defect, respectively. The width of the WG within the lattice is $\mathrm{w}_{\mathrm{g}}=4 \mu \mathrm{m}$ and the distance between neighboring WGs is $s=4 \mu \mathrm{m}$. ND is fixed in the middle of the lattice and its position does not change, whereas the position of LD is changeable. The nonlinearity strength of ND has been taken to be $\Gamma=1.3$ or 10 .

Since the aim of this research is to investigate the common influence of the LD and ND on the beam propagation, we will start with analyzing the case in which the LD is placed next to the ND, in the first WG to the right of it. The width of LD has been varied as well as the values of nonlinearity of the ND. If regime possesses $10 \%$ of the amplitude value we have claimed that regime existed. In Figs. 2-7. amplitude of refractive index of modulation has larger values on narrow LD than in the case of wider. Linear part of refractive index of modulation is highly dependent of geometry of the system. Hence, changing of the width of WGs or the width of the separation between WGs, will lead to the formation of different amplitude of refractive index. It is shown, by number of numerical simulations, that narrow LD forms larger value of amplitude of refractive index of modulation than wider one.

It can be seen that in this case when the light beam is launched into the ND, the width of LD influences the localization of the light on the ND, Figure $2 \mathrm{a}$. Namely, the narrow LD is better potential barrier than the wide one and the light beam is localized completely on the ND. The efficiency of capturing the beam depends on the values of nonlinearity and in the case of higher nonlinearity, capturing effect is very weak, almost not noticeable (Fig.2c). In the case when the LD $6 \mu \mathrm{m}$ wide is placed next to the ND in which the beam is initiated breathing mode between two neighbouring WGs is formed. One component of the breathing mode is placed at the nonlinear WG whereas the other is located at the adjacent LD, Fig.2b. For higher nonlinearity strength one can observe light scattering and absence of energy localization, Fig.2d.
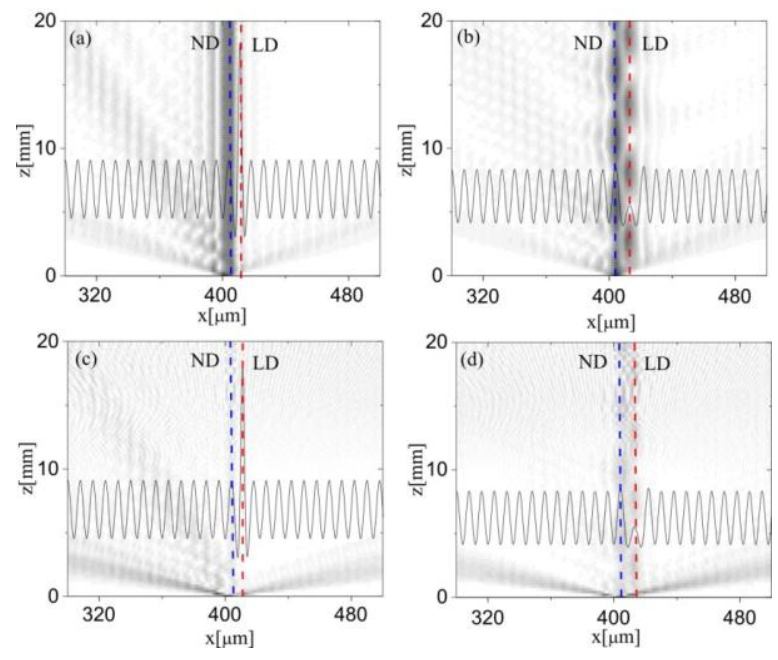

Fig. 2. 2D plot of the average beam intensity profiles, $\mathrm{LD}$ is located at the $1^{\text {st }} \mathrm{wg}$ on the right of the ND, incident beam enters the ND: (a) nonlinearity strength of ND is $\Gamma=1.3$, width of LD is $2 \mu \mathrm{m}$; (b) $\Gamma=1.3$, width of LD is $6 \mu \mathrm{m}$; (c) $\Gamma=10$, width of LD is $2 \mu \mathrm{m}$; (d) $\Gamma=10$, width of LD is $6 \mu \mathrm{m}$. Blue line shows the position of the nonlinear defect, while the red line denotes the position of the linear defect.

If the beam is inserted into the LD which is next to the ND, one can observe that the strength of nonlinearity have a small influence on the beam propagation in the LD, Fig. 3. The width of LD has the dominant influence on the beam propagation. When the beam enters the narrow LD positioned next to the $\mathrm{ND}$, the beam is strongly localized at the position of LD regardless of the strength of ND, (Figs. 3a,c).
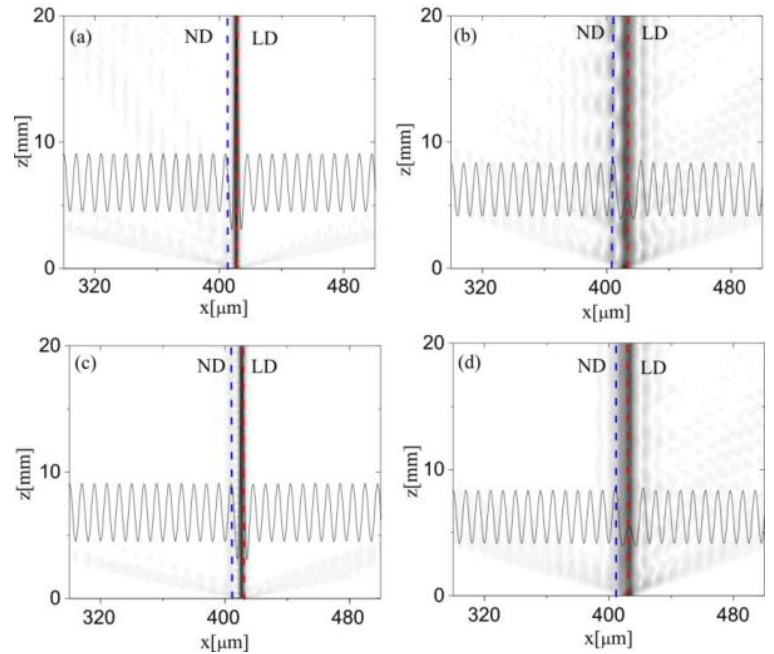

Fig. 3. 2D plot of the average beam intensity profiles, $\mathrm{LD}$ is located at the $1^{\text {st }} \mathrm{wg}$ on the right of the ND, incident beam enters the LD: (a) $\Gamma=1.3$, LD is $2 \mu \mathrm{m}$ wide; (b) $\Gamma=1.3$, width of LD is $6 \mu \mathrm{m}$; (c) $\Gamma=10$, width of LD is $2 \mu \mathrm{m}$; (d) $\Gamma=10$, width of LD is $6 \mu \mathrm{m}$. Blue line shows the position of the nonlinear defect, while the red line denotes the position of the linear defect. 
If the beam is launched into the wide LD (Fig. 3b), localized mode has a smaller amplitude than in the case when the light has been inserted into the narrow LD. Here, one can see that higher nonlinearity strength of the ND (Fig. 3d) will slightly improve localization on LD.

We have investigated the influence of the transverse kick of the input beam on its propagation through the system. For lower values of the kick $(\alpha \leq$ $2 \pi / 30$ ), if the beam is launched from the left towards the ND ( $\Gamma=1.3)$ with LD $2 \mu \mathrm{m}$ wide placed next to it, on the side opposite to the input beam, it is possible to obtain mild capturing of the beam at the position of ND and partial reflection from it (Figure 4a). For a wide LD, this capturing at the ND has not been observed, Figure 4b. For narrow LD and higher nonlinearity $(\Gamma=10)$ beam launching at same position (left towards ND) leads towards beam capturing at ND position. However, for wide LD and same nonlinearity $(\Gamma=10)$ reflection of the light at position left of ND is dominant process. Light reflection becomes dominant process no matter from which side (left from ND or right from $\mathrm{LD}$ ) the input beam is launched into the lattice.
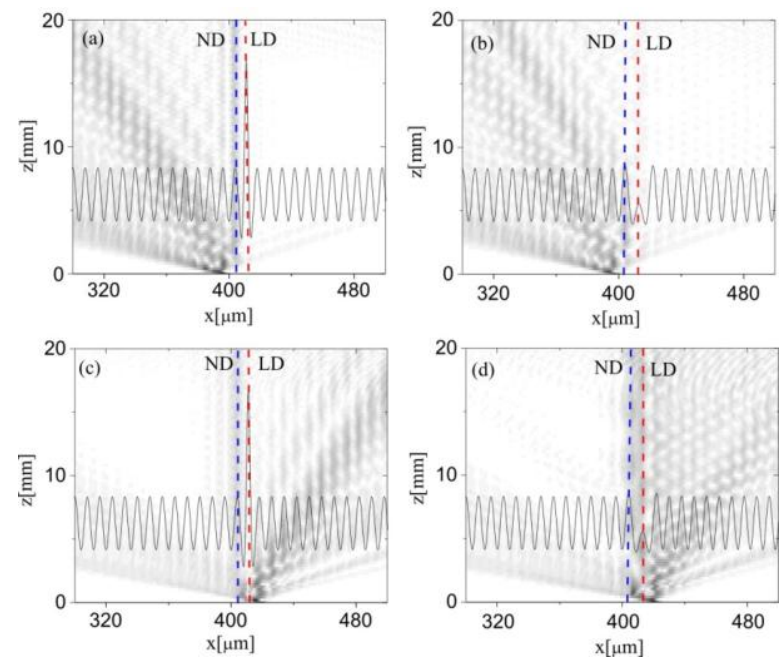

Fig.4 2D plot of the average beam intensity profiles, $\mathrm{LD}$ is located at the $1^{\mathrm{st}} \mathrm{wg}$ on the right of the ND, $\alpha=2 \pi / 30$ : (a) incident beam enters the $1^{\text {st }} \mathrm{wg}$ on the left of the ND, $\Gamma=1.3$, width of LD is $2 \mu \mathrm{m}$; (b) width of LD is $6 \mu \mathrm{m}, \Gamma=1.3$; (c) incident beam enters the $1^{\text {st }} \mathrm{wg}$ on the right of the $\operatorname{LD}, \Gamma=10$, width of $\mathrm{LD}$ is $2 \mu \mathrm{m}$; (d) width of LD is $6 \mu \mathrm{m}, \Gamma=10$. Vertical blue dashed line denotes the ND, while vertical red line shows LD.

For nonlinearity strength $\Gamma=10$ and the same low values of the kick when the beam is launched towards the narrow LD, weak capturing at the position of the LD has been obtained, Figure 4c. Greater width of LD contributes to more efficient trapping of the beam at the LD, Figure 4d. Partial reflection is visible here as well. The higher value of the transverse kick leads to the decrease of the reflection and mild capturing of energy at the ND, transmission begins to appear as well. Transmission, in the presence of the kicks that are big enough $(2 \pi / 6$ to $2 \pi / 12)$, becomes the only effect which appears for the given values of the parameters. When defects are more distant and with beam launched in the cavity that's placed between ND and LD, incidence angle of the beam affects its capturing and its zig-zag modes. Cavity is the formed area between ND and LD, when they are separated by at least one WG. If the beam is launched outside the cavity, light reflection becomes dominant process.

The increase of the distance between the defects leads to the cavity formation in which the localization of energy can appear. This is illustrated in Figures 5a, b, $c$ where the LD is located at the second WG on right of the ND. Trapping of the light is observed when the light is launched into the ND, Fig. 5a. When the beam is launched in the wg between the two defects, i. e. in the cavity, it becomes captured in it and this is due to potential barriers which appear as a consequence of defects' presence on the right and left side, it can capture the light efficiently now, Figures 5b, c. By comparing these two figures it can be concluded that the width of the LD has no impact on the efficiency of capturing the light in the cavity. Efficiency of capturing means the ratio between the amplitude of the light, captured within the region between the defects or at the positions of the defects, and amplitude of the input light beam. Since the effects of only linear and only ND on the beam propagation through the lattice have already been investigated (Kuzmanović et al. 2015a), by comparing those results with ones obtained here, it can be seen that capturing of the light in the LD located at the second WG from nonlinear one is equivalent to the one obtained when only LD has been investigated, Figure 5d, e. The light of capturing in the LD has been observed for narrow and wide LD, when the light beam has been launched into the LD. ND has a small influence on the formation of localized mode.

For greater distances between the ND and LD, it is possible to obtain multicomponent breathing modes in the cavity. In Figure 6, two-component breathing modes and the influence of the closeness of either of the two defects on these modes have been shown.There are no significant qualitative nor quantitative differences on the light beam propagation whether the light beam is launched near the narrow LD or near the wide LD within the cavity, Figures $6 \mathrm{a}$ and

\section{Physics}


6c. In both cases, two-component breathing mode is formed in the cavity between the defects. However, if we replace the narrow $\mathrm{LD}$ with the wide one, the two cases will be slightly different, Figures $6 \mathrm{~b}$ and $6 \mathrm{~d}$. In this case, the breathing-mode component closer to the wide LD (the beam is launched in the cavity, in the WG adjacent to the LD) will be more pronounced than the one adjacent to the ND.

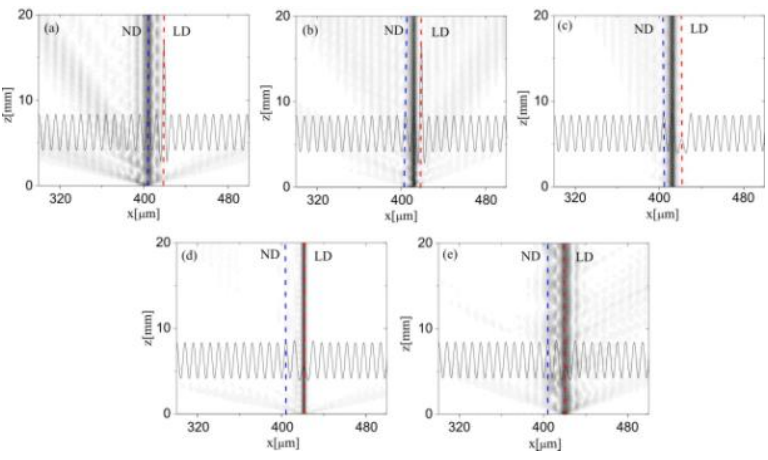

Fig. 5. 2D plot of the average beam intensity profiles, LD is located at the $2^{\text {nd }} \mathrm{wg}$ on the right of the ND: (a) $\Gamma=1.3$, incident beam enters the ND, width of LD is 2 $\mu \mathrm{m}$;(b) $\Gamma=10$, incident beam enters the $1^{\text {st }}$ on the right of the ND, width of LD is $2 \mu \mathrm{m}$ (c) $\Gamma=10$, incident beam enters the $1^{\text {st }}$ on the right of the ND, width of LD is $6 \mu \mathrm{m}$, (d) $\Gamma=10$, incident beam enters the LD $2 \mu \mathrm{m}$ wide; (e) $\Gamma=10$, incident beam enters the LD $6 \mu \mathrm{m}$ wide. Vertical blue dashed line denotes the ND, while vertical red line shows LD.
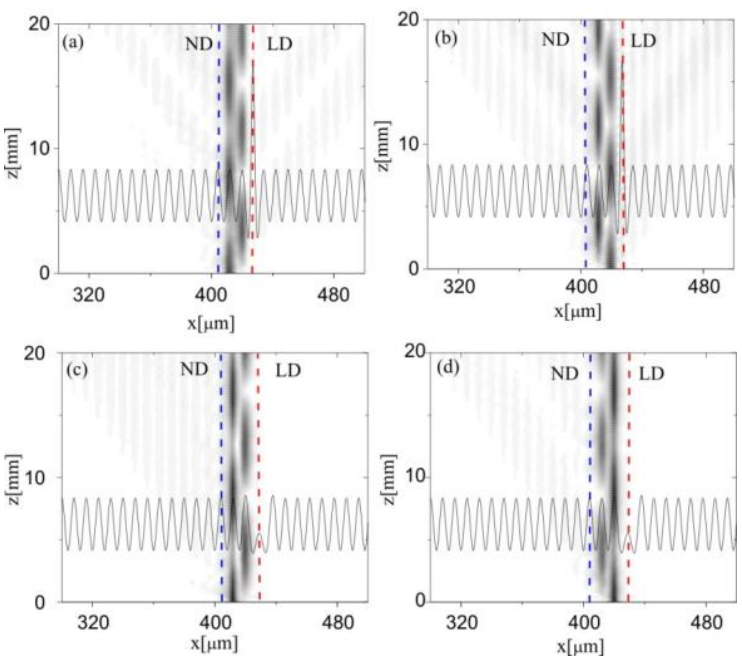

Fig. 6. 2D plot of the average beam intensity profiles, $\Gamma=10$, LD is located at the $3^{\text {rd }} \mathrm{wg}$ on the right of the ND: (a) incident beam enters the $1^{\text {st }} \mathrm{wg}$ on the right of the ND, width of LD is $2 \mu \mathrm{m}$; (b) incident beam enters the $2^{\text {nd }} \mathrm{wg}$ on the right of the ND, width of LD is 2 $\mu \mathrm{m}$; (c) incident beam enters the $1^{\text {st }} \mathrm{wg}$ on the right of the ND, width of LD is $6 \mu \mathrm{m}$; (d) incident beam enters the $2^{\text {nd }} \mathrm{wg}$ on the right of the ND, width of LD is 6 $\mu \mathrm{m}$. Vertical blue dashed line denotes the ND, while vertical red line shows LD.
In the area between the ND and narrow LD, if the light is launched in the middle of the cavity it is possible to obtain symmetric - like structures because at the first sight they look symmetric but further analysis shows that they not, Figures 7, a, b. For smaller distances between the defects (three WGs or $28 \mu \mathrm{m}$ ) (Figure 7a), there is a dominant central mode whereas for greater distances, this central mode is no longer dominant (Figure 7b, d). Central mode is the one located at the center of the distance between the LD and ND. In the case of the LD $6 \mu \mathrm{m}$ wide, it is not possible to obtain symmetrical structures irrespective of the distance between defects, Figure 7e. This means that the potential of the wide LD cannot balance the potential of ND, thus enabling the formation of symmetrical structure. It is possible to obtain zig-zag modes as well, when the beam is launched at the WG near the one or the other defect, Figure $7 \mathrm{c}$.
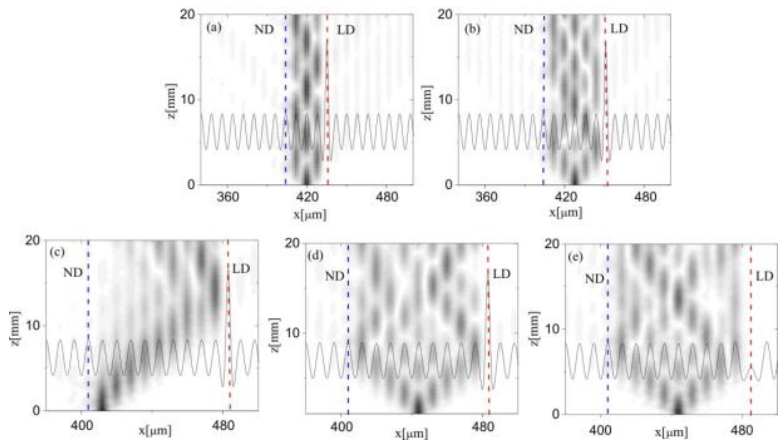

Fig. 7. 2D plot of the average beam intensity profiles, $\Gamma=10$, width of LD is $2 \mu \mathrm{m}$ : (a) incident beam enters the $2^{\text {nd }} \mathrm{wg}, \mathrm{LD}$ is located at the $4^{\text {th }} \mathrm{wg}$; (b) incident beam enters the $3^{\text {rd }} \mathrm{wg}, \mathrm{LD}$ is located at the $6^{\text {th }} \mathrm{wg}$; (c) incident beam enters the $1^{\text {st }} \mathrm{wg}, \mathrm{LD}$ is located at the $10^{\text {th }} \mathrm{wg}$; (d) incident beam enters the $5^{\text {th }} \mathrm{wg}$, LD is located at the $10^{\text {th }} \mathrm{wg}$; (e) incident beam enters the $5^{\text {th }}$ wg, LD $6 \mu \mathrm{m}$ wide is located at the $10^{\text {th }} \mathrm{wg}$ on the right of the ND. Vertical blue dashed line denotes the ND, while vertical red line shows LD.

\section{CONCLUSION}

The influence of ND and LD on the light beam propagation through 1D PL is investigated numerically. LD represents a WG which is narrower or wider than the rest of the WGs in the array. For narrow $\mathrm{LD}$, when the beam is launched without the transverse kick, the total localization of the light on either of the defects has been observed. The narrow LD is more efficient in the localization of the light at the ND when they are located next to each other. On the other hand, the significant influence of ND on the beam 
propagation in this system is not observed. The localization of the light is stronger at the narrow LD and for smaller nonlinearity strength. The influence of ND and LD on the light propagation in the area between them has also been investigated. Changing the initial parameters such as the initial position of the light beam, incident angle, strength of ND and the width of LD, different regimes of light propagation have been identified: localized modes, symmetrical breathing structures and symmetric - like zig-zag modes. The results presented here can be useful for all-optical control of the wave transmission in interferometry.

\section{REFERENCES}

Beličev, P., Ilić, I., Stepić, M., Maluckov, A., Tan, Y., \& Chen, F. 2010. Observation of linear and nonlinear strongly localized modes at phase-slip defects in one-dimensional photonic lattices. Opt. Letters, 35, pp. 3099-3101.

Chen, F., Stepić, M., Rüter, C., Runde, D., Kip, D., Shandarov, V., . . . Segev, M. 2005. Discrete diffraction and spatial gap solitons in photovoltaic LiNbO3 waveguide arrays. Opt Express, 13(11), pp. 4314-24. pmid:19495346. doi:10.1364/OPEX.13.004314.

Christodoulides, D.N., \& Joseph, R.I. 1988. Discrete self-focusing in nonlinear arrays of coupled waveguides. Opt. Letters, 13, pp. 794-796.

Denz, C., Flach, S., \& Kivshar, Y.S. 2010. Nonlinearities in periodic structures and metamaterials. Springer Series In Optical Sciences, Springer-Verlag Berlin Heidelberg, 150.

Fedele, F., Yang, J.K., \& Chen, Z.G. 2005. Defect modes in one-dimensional photonic lattices. Opt. Letters, 30, pp. 1506-1508.

Kuzmanović, S., Stojanović-Krasić M., , Milović, D., Radosavljević, A., Gligorić, G., Maluckov, A., \& Stepić, M. 2015. Defect induced wave-packet dynamics in linear one-dimensional photonic lattices. Phys. Scripta, 90, p. 25505.

Kuzmanović, S., Stojanović-Krasić, M., Milović, D., Miletić, M., Radosavljević, A., Gligorić, G., . . Stepić, M. 2015. Light propagation inside 'cavity' formed between nonlinear defect and interface of two dissimilar one-dimensional linear photonic lattices. Eur. Phys. J. D, 69, p. 207.

\footnotetext{
*E-mail: slavica.kuzmanović@pr.ac.rs
}

Lahini, Y., Avidan, A., Pozzi, F., Sorel, M., Morandotti, R., Christodoulides, D.N., \& Silberberg, Y. 2008. Anderson localization and nonlinearity in one-dimensional disordered photonic lattices. Phys. Rev. Letters, 100, p. 13906.

Meier, J., Stegeman, I.G., Christodoulides, D.N., Silberberg, Y., Morandotti, R., Yang, H., . . . Aitchison, J.S. 2005. Beam interactions with a blocker soliton in one-dimensional arrays. Opt. Letters, 30, pp. 1027-1029.

Miroshnichenko, A.E., Flach, S., \& Kivshar, S.(. 2010. Fano resonances in nanoscale structure, Rev. Mod. Phys., 82, pp. 2257-2298.

Morandotti, R., Eisenberg, H.S., Mandelik, D., Silberberg, Y., Modotto, D., Sorel, D., . . . Aitchison, J.S. 2003. Interactions of discrete solitons with structural defects. Opt. Letters, 28, pp. 834-836.

Molina, M., \& Kivshar, S.(. 2008. Nonlinear localized modes at phase-slip defects in waveguide arrays. Opt. Letters, 33, pp. 917-919.

Noda, S., Fujita, M., \& Asano, T. 2007. Spontaneousemission control by photonic crystals and nanocavities. Nature Photonics, 1(8), pp. 449-458. doi:10.1038/nphoton.2007.141

Naether, U., Rivas, D.E., Larenas, M.A., Molina, M.I., \& Vicencio, R.A. 2009. Fano resonances in waveguide arrays with saturable nonlinearity. Opt. Letters, 34(2721)

Radosavljević, A., Gligorić, G., Maluckov, A., \& Stepić, M. 2014. Control of light propagation in onedimensional quasi-periodic nonlinear photonic lattices. J. Optics, 16(025201).

Schwartz, T., Bartal, G., Fishman, S., \& Segev, M. 2007. Transport and Anderson localization in disordered two-dimensional photonic lattices. Nature, 446(7131), pp. 52-5. pmid:17330037. doi:10.1038/nature05623.

Smirnov, E., Stepić, M., Rüter, C.E., Kip, D., \& Shandarov, V. 2006. Observation of staggered surface solitary waves in one-dimensional waveguide arrays. Opt. Letters, 31, pp. 2338-2340.

Stojanović-Krasić, M., Mančić, A., Kuzmanović, S., Veljković-Đorić, S., \& Stepić, M. 2016. Linear and interface defects in composite linear photonic lattice. submitted to Optics Communications.

Tran, P. 1997. Optical limiting and switching of short pulses by use of a nonlinear photonic bandgap structure with a defect. J. Opt. Soc. Am. B, 14, pp. 2589-2595. 